\title{
Achieving three-peak white organic light-emitting devices using wavelength-selective mirror electrodes
}

\author{
Yin-Jui Lu, ${ }^{1}$ Chih-Hao Chang, ${ }^{1}$ Chun-Liang Lin, ${ }^{1}$ Chung-Chih Wu, ${ }^{1, a)}$ Hsiang-Lun Hsu, ${ }^{2}$ \\ Liang-Jyi Chen, ${ }^{2}$ Yu-Ting Lin, ${ }^{2}$ and Ryuji Nishikawa ${ }^{2}$ \\ ${ }^{1}$ Department of Electrical Engineering, Graduate Institute of Photonics and Optoelectronics, \\ and Graduate Institute of Electronics Engineering, National Taiwan University, Taipei, Taiwan 10617, \\ Republic of China \\ ${ }^{2}$ TPO Displays Corp., Miao-Li, Taiwan 350, Republic of China
}

(Received 29 October 2007; accepted 13 February 2008; published online 28 March 2008)

\begin{abstract}
We report an effective approach based on wavelength-selective mirrors for implementing three-peak white-emitting organic light-emitting devices (OLEDs). Such three-peak white OLEDs have electroluminescence spectra matching better with the transmission spectra of typical color filters and thus give much enhanced color gamut for full-color OLED display applications. The wavelength-selective mirror uses the metal/dielectric stack that is compatible with the OLED fabrication. (C) 2008 American Institute of Physics. [DOI: 10.1063/1.2890847]
\end{abstract}

White organic light-emitting devices (WOLEDs) have attracted much attention due to their applications in displays and lighting. ${ }^{1-5}$ For full-color display applications, the combination of WOLEDs and color filters is one of the most promising technologies since it avoids the precise shadow masking often used in the separate red-green-blue (RGB) pixilation, ${ }^{3,4}$ and thus is beneficial to realizations of higher display resolutions and large-area scale-up. WOLEDs, ${ }^{1-5}$ usually exhibit rather broad electroluminescence (EL) spectra. In addition, the transmission spectra of most available color filters are also broad and match poorly with the EL spectra of WOLEDs. ${ }^{6}$ As a result, RGB colors filtered from WOLEDs are often less saturated than those achieved with the separate RGB pixilation. 3,4

In 2004, Sony demonstrated a full-color active-matrix OLED display with saturated RGB colors by combination of the white emitter, the microcavity structure, and color filters. ${ }^{4}$ In such a configuration, individual RGB colors was created by placing the same WOLEDs into microcavities having resonant wavelengths set at corresponding primary colors; color filters were used to further purify emission colors. The tuning of the resonant wavelength (and the cavity length) was accomplished by depositing and patterning the transparent indium tin oxide (ITO) layers of different thicknesses in RGB subpixels. Depositing and patterning ITO into different thicknesses, however, highly complicates the fabrication and mass production. In view of these, WOLEDs with three narrow emission peaks occurring at desired RGB wavelengths and well matching spectra of RGB color filters are highly desired. In this work, a simple and effective approach is developed to accomplish the three-peak WOLED, which adopts the wavelength-selective mirror as the cathode and the reflecting mirror. Such a wavelength-selective mirror is compatible with the OLED deposition and yields a WOLED EL spectrum giving more saturated RGB colors when combined with color filters.

Fig. 1shows the structure of the conventional bottomemitting OLED. In such structure, the directly out-going emission interferes with the emission reflected from the metal electrode, determining the out-coupled emission spec-

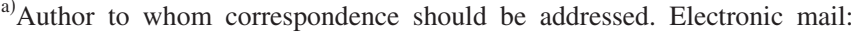
chungwu@cc.ee.ntu.edu.tw. trum and intensity. ${ }^{7-9}$ In order to obtain constructive interference and thus maximize the out-coupled intensity, it roughly requires the location of emitters to the metal electrode be consistent with the antinode condition for major emission wavelengths (i.e., the emitter-to-metal round-trip phase change $\phi(\lambda)$ equals multiple integers of $2 \pi) .^{7-9}$ In referring to Fig. 1, this corresponds to $\phi(\lambda)=-2 k L+\theta(\lambda)=2 m \pi$, where $k$ is the wavevector (taking into consideration the refractive indices of organic layers), $L$ is the emitter-to-mirror distance, $\lambda$ is the wavelength, and $\theta(\lambda)$ is phase change associated with the mirror reflection (having a reflection coefficient of $r e^{j \theta(\lambda)}$, Fig. 1). Based on such a principle, to achieve a three-peak WOLED showing EL peaks at desired red, green, and blue wavelengths and deep minima at wavelengths in between, one shall modify the phase property of the reflective mirror such that the directly out-going emission constructively interferes with the reflected emission at desired red, green, and blue wavelengths (i.e., satisfy the antinode condition) and destructively interferes at wavelengths in between (i.e., the node condition). Furthermore, the reflectance of this mirror must be high enough over the whole visible range since the degree of constructive and destructive interference highly depends on the intensity of the reflected light relative to that of the directly emitting light.

Such wavelength-selective mirror could be realized by stacking dielectric materials and thin metal layers. Owing to the large difference between their refractive indices, multiple-beam interference would occur within the mirror, and a strongly wavelength-dependent phase change could be induced. For instance, a structure such as thin metal/dielectric/thin metal/dielectric/thick metal can be

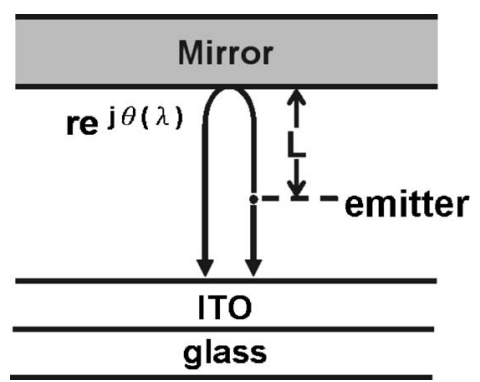

FIG. 1. Schematic diagram of the conventional OLED structure. 

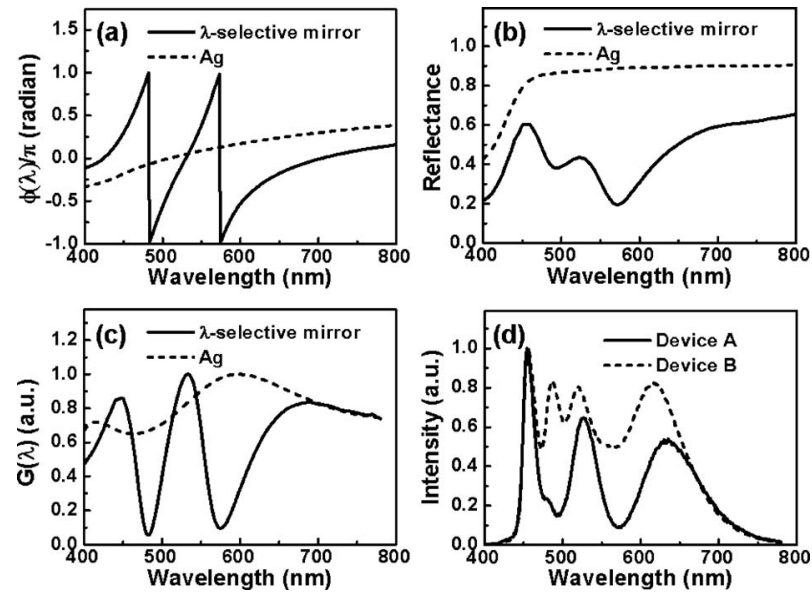

FIG. 2. (a) Calculated emitter-to-mirror round-trip phase changes $\phi(\lambda)$, (b) calculated reflectance spectra, and (c) intensity modification factors $G(\lambda)$ seen by an emitter placed $50 \mathrm{~nm}$ in front of the designed wavelengthselective mirror and a thick silver mirror. (d) EL spectra of WOLED A and $\mathrm{B}$ in the forward direction at a driving current of $10 \mathrm{~mA} / \mathrm{cm}^{2}$.

used to implement the phase properties described above. Figure 2(a) shows the calculated round-trip phase changes [i.e., $\phi(\lambda)=-2 k L+\theta(\lambda)]$ for an emitter placed $50 \mathrm{~nm}$ [assuming an optical background of the typical organic electrontransport material tris(8-hydroxyquinoline)aluminum $\left.\left(\mathrm{Alq}_{3}\right)\right]$ in front of a composite mirror $\left[\mathrm{Ag}(8 \mathrm{~nm}) / \mathrm{Alq}_{3}(90 \mathrm{~nm}) / \mathrm{Ag}\right.$ $\left.(26 \mathrm{~nm}) / \mathrm{Alq}_{3}(100 \mathrm{~nm}) / \mathrm{Ag}(150 \mathrm{~nm})\right]$ and a typical thick $\mathrm{Ag}$ mirror. Here, the first thin metal layer can be used as the cathode electrode for the OLED. $\mathrm{Alq}_{3}$ is used as the dielectric material here due to its high transparency in the visible range and compatibility with the OLED fabrication, ${ }^{10}$ but other transparent dielectric materials (conducting, semiconducting, or insulating) could be similarly used. Ag is used as the metal layer due to its lower absorption loss in the visible range and highest conductivity among electrode materials. As shown in Fig. 2(a), by carefully designing layer thicknesses of the composite mirror, constructive interference occurs at wavelengths around 424, 530, and $700 \mathrm{~nm}$ (i.e., at red, green, and blue wavelengths), while the destructive interference occurs at the wavelengths around 482 and $574 \mathrm{~nm}$. In contrast, the round-trip phase change associated with the thick Ag electrode varies only gradually with the wavelength, not possibly giving constructive/destructive interference at multiple wavelengths.

Figure 2(b) compares the calculated reflectance spectra of the typical Ag mirror and the present composite mirror. The reflectance of the composite mirror is lower, yet around the wavelengths of constructive interference the reflectance remains around 50\%-60\%. Finally, considering the optical properties of mirror electrodes, Fig. 2(c) shows the intensity modification factor $G(\lambda)$ as a function of the wavelength that would act to modify/modulate the out-coupled emission spectra of an emitter (located $50 \mathrm{~nm}$ in front of the mirror). $G(\lambda)$ was calculated using the rigorous electromagnetic modeling of the OLED emission that had been developed previously, ${ }^{8-13}$ which is based on the equivalence between the emission from a molecular dipole transition and the radiation from a classical electrical dipole antenna. Apparently, with such a spectral modification function, the EL spectrum of a WOLED shall be effectively shaped to achieve the de-

sired three-peak spectrum. trode on top of the same organic stack (device A), the white
Downloaded 18 Feb 2009 to 140.112.113.225. Redistribution subject to AlP license or copyright; see http://apl.aip.org/apl/copyright.jsp

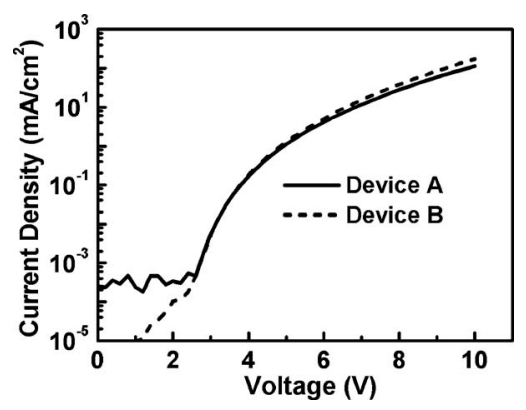

FIG. 3. Current-voltage characteristics of devices A and B.

A three-peak WOLED (device A) and a control WOLED (device B) utilizing the wavelengthselective mirror and the thick $\mathrm{Ag}$ as the top electrodes, respectively, were fabricated and tested. Device A had the structure of glass/ITO $(120 \mathrm{~nm}) / m$-MTDATA $\quad(30 \mathrm{~nm}) / \alpha$-NPD $\quad(20 \mathrm{~nm}) /$ ADN:perylene $\quad(1 \mathrm{wt} \%, 7.5 \mathrm{~nm}) / \mathrm{Alq}_{3}: \mathrm{C} 545 \mathrm{~T}$ (1 wt \%,5 nm)/ $\mathrm{Alq}_{3}$ :DCJTB $\quad(0.7 \mathrm{wt} \%, 7.5 \mathrm{~nm}) / \mathrm{Alq}_{3}$ $(40 \mathrm{~nm}) / \mathrm{LiF} \quad(0.5 \mathrm{~nm}) / \mathrm{Al} \quad(1 \mathrm{~nm}) / \mathrm{Ag} \quad(8 \mathrm{~nm}) / \mathrm{Alq}_{3}$ $(90 \mathrm{~nm}) / \mathrm{Ag}(26 \mathrm{~nm}) / \mathrm{Alq}_{3}(100 \mathrm{~nm}) / \mathrm{Ag}(150 \mathrm{~nm})$. Device $B$ had the structure of glass/ITO $(120 \mathrm{~nm}) / \mathrm{m}$ MTDATA $\quad(30 \mathrm{~nm}) / \alpha$-NPD $\quad(20 \mathrm{~nm}) / \mathrm{ADN}$ :perylene (1 wt $\%, 7.5 \mathrm{~nm}) / \mathrm{Alq}_{3}: \mathrm{C} 545 \mathrm{~T}$

(1 wt \%,5 nm)/ $\mathrm{Alq}_{3}$ :DCJTB $\quad(0.7 \mathrm{wt} \%, 7.5 \mathrm{~nm}) / \mathrm{Alq}_{3} \quad(40 \mathrm{~nm}) / \mathrm{LiF}$ $(0.5 \mathrm{~nm}) / \mathrm{Al}(1 \mathrm{~nm}) / \mathrm{Ag}(150 \mathrm{~nm}) . \quad$ In both devices, $4,4^{\prime}, 4^{\prime \prime}$-tris $(N$-3-methylphenyl- $N$-phenyl-amino) triphenylamine $\quad\left(m\right.$-MTDATA), $\quad N, N^{\prime}$-bis(naphthalen-1-yl)$N, N^{\prime}$-bis(phenyl)-benzidine ( $\alpha$-NPD) and $\mathrm{Alq}_{3}$ were used as the hole-injection, hole-transport, and electron-transport layers, respectively. ${ }^{11}$ Thin $\mathrm{LiF} / \mathrm{Al}$ was used as the electron-injection layer to enhance electron injection from the Ag cathode. ${ }^{9-11}$ Multiple emission layers (EMLs) were used for generating white emission, which consisted of 9,10-di-(2-naphthyl)anthracene $(\mathrm{ADN})$ doped with perylene as the blue EML, ${ }^{14} \mathrm{Alq}_{3}$ doped with 10-(2-benzothiazolyl)2,3,6,7- tetrahydro- $\quad 1,1,7,7,-\quad$ tetramethyl$1 H, 5 H, 11 H$-(1) - benzopyropyrano-(6,7-8-i,j)quinolizin-11one $(\mathrm{C} 545 \mathrm{~T})$ as the green EML, ${ }^{8,10}$ and $\mathrm{Alq}_{3}$ doped with 4-(dicyanomethylene) - 2 - $t$-butyl-6-(1,1,7,7-tetramethyljulolidyl- 9-enyl)-4H-pyran (DCJTB) as the red EML. ${ }^{15}$ Their thicknesses and doping concentrations were adjusted to achieve white emission. All organic, LiF, and metal layers were deposited consecutively by thermal evaporation under a base pressure of $\sim 10^{-6}$ torr without breaking vacuum. The present WOLEDs containing three emitting layers show satisfactory enough white color at the practical brightnesses of $100-1000 \mathrm{~cd} / \mathrm{m}^{2}$.

Figure 2(d) shows the EL spectra of devices A and B in the forward direction at a current density of $10 \mathrm{~mA} / \mathrm{cm}^{2}$ (giving brightnesses of 235 and $477 \mathrm{~cd} / \mathrm{m}^{2}$ for $\mathrm{A}$ and $\mathrm{B}$, respectively). Figure 3 compares the current-voltage $(I-V)$ characteristics of both devices; the adoption of the wavelength-selective mirror electrode does not alter the electrical characteristics of devices. The control device (device B) exhibits a broadband white emission with CIE (Commission Internationale de l'Eclairage) coordinates of (0.359, 0.374), covering wavelengths from 440 to $700 \mathrm{~nm}$ and showing peaks at the wavelengths of 455, 488, 520, and $615 \mathrm{~nm}$. By applying the wavelength-selective mirror electrode on top of the same organic stack (device A), the white AIP license or copyright; see http://apl.aip.org/apl/copyright.jsp 

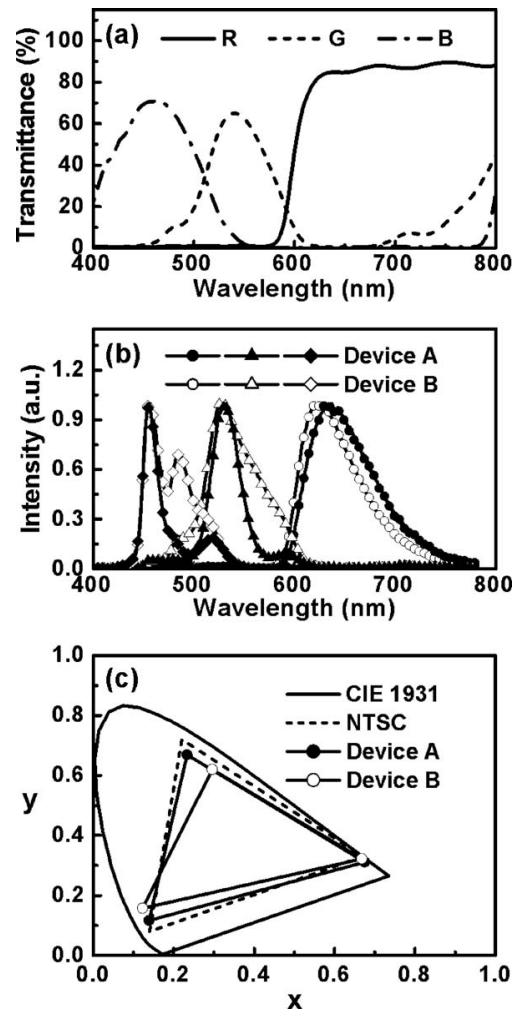

FIG. 4. (a) Transmission spectra of micrometer-thick RGB color filters coated on the glass substrate, (b) filtered R (circle), G (triangle), and B (diamond) spectra obtained by passing emission of both WOLEDs through the RGB color filters. (c) $1931 \mathrm{CIE}$ coordinates of filtered RGB colors for devices A and B in comparison with the NTSC standard.

emission still has satisfactory CIE coordinates of $(0.323$, 0.330 ), yet the spectrum now shows three distinct peaks at the saturated RGB wavelengths of 454,527 , and $635 \mathrm{~nm}$ and two deep minima in between (492 and $574 \mathrm{~nm}$ ). This demonstrates the effectiveness of the wavelength-selective mirror in shaping the EL spectra of WOLEDs. It is noticed that the peak and minimum wavelengths of the three-peak WOLED do not exactly match the constructive/destructive interference conditions in Fig. 2, mainly associated with the influences of the intrinsic emission spectrum of the emitters. The EL peaks of the three-peak WOLED also show a slight blueshift with viewing angles due to angular dependence of $\phi(\lambda)$, yet such a shift is mitigated in the filtered RGB colors due to the effects of color filters.

When combined with the color filters typically used in display industries, the present three-peak WOLED gives a substantially enhanced color gamut. Figure 4(a) shows the transmission spectra of micrometer-thick RGB color filters coated on the glass substrate. In Fig. 4(a), one notices the rather broad transmission spectra and the significant overlaps between colors, both of which would degrade the color purity of filtered colors. Figure 4(b) shows the filtered RGB spectra obtained by passing emission of both WOLEDs through color filters. With the EL spectrum matching better the transmission spectra of the color filters, the three-peak WOLED (device A) gives sharper and more saturated colors than the conventional WOLED (device B). CIE coordinates of filtered RGB colors for both WOLEDs are shown in Fig. 4(c) for comparison. By using wavelength-selective mirror to implement the three-peak spectrum, device A gives a greatly enhanced color gamut [relative to the NTSC (National Television Standards Committee) standard] of $87 \%$ (versus $70 \%$ of device B).

The efficiency of the present three-peak WOLED (A) is lower than that of the conventional WOLED (B). At the current density of $10 \mathrm{~mA} / \mathrm{cm}^{2}$, the current efficiency of the three-peak WOLED (A) is about $50 \%$ of the control device (B) efficiency ( $2.4 \mathrm{vs} 4.8 \mathrm{~cd} / \mathrm{A}$ ), while the external quantum efficiency of device A is about $70 \%$ of the device B efficiency ( $2 \%$ vs $2.8 \%$ ). This is mainly because in the present three-peak WOLED, part of emission is suppressed by destructive interference and because the wavelength-selective mirror exhibits a relatively lower reflectance than the typical metal mirror. In the future, the efficiency (and even color performances) of the three-peak WOLED shall be possibly further improved by better designs of the wavelengthselective mirrors (e.g., raising the reflectance). In addition, it is interesting to notice that owing to the better match between the EL spectrum of the three-peak WOLED and the transmission of color filters, the efficiency loss caused by color filters is relatively lower, compared to the case of the conventional WOLED.

In summary, we have developed an effective approach based on wavelength-selective mirrors for implementing three-peak WOLEDs. Such three-peak WOLEDs have EL spectra matching better with transmission spectra of typical color filters and thus give much enhanced color gamuts for full-color OLED displays.

The authors would like to acknowledge financial support from National Science Council of Republic of China and TPO Display Group.

${ }^{1}$ J. Kido, M. Kimura, and K. Nagai, Science 267, 1332 (1995).

${ }^{2}$ B. W. D'Andrade and S. R. Forrest, Adv. Mater. (Weinheim, Ger.) 16, 1585 (2004).

${ }^{3}$ J. P. Spindler, T. K. Hatwar, M. E. Miller, A. D. Arnold, M. J. Murdoch, P. J. Kane, J. E. Ludwicki, and S. A. Van Slyke, Society for Information Display (SID) International Symposium, Digest of Technical Papers, 2005, p. 36.

${ }^{4}$ M. Kashiwabara, K. Hanawa, R. Asaki, I. Kobori, R. Matsuura, H. Yamada, T. Yamamoto, A. Ozawa, Y. Sato, S. Terada, J. Yamada, T. Sasaoka, S. Tamura, and T. Urabe, Society for Information Display (SID) International Symposium, Digest of Technical Papers, 2004, p. 1017.

${ }^{5}$ A. Dodabalapur, L. J. Rothberg, R. H. Jordan, T. M. Miller, R. E. Slusher, and J. M. Phillips, J. Appl. Phys. 80, 6954 (1996).

${ }^{6}$ Liquid Crystal Displays: Addressing Schemes and Electro-Optical Effects, edited by E. Lueder (Wiley, New York, 2001), pp. 289-295.

${ }^{7}$ S. K. So, W. K. Choi, L. M. Leung, and K. Neyts, Appl. Phys. Lett. 74, 1939 (1999).

${ }^{8}$ C.-L. Lin, T.-Y. Cho, C.-H. Chang, and C.-C. Wu, Appl. Phys. Lett. 88, 081114 (2006)

${ }^{9}$ C.-L. Lin, H.-W. Lin, and C.-C. Wu, Appl. Phys. Lett. 87, 021101 (2005).

${ }^{10}$ C.-L. Lin, H.-C. Chang, K.-C. Tien, and C.-C. Wu, Appl. Phys. Lett. 90, 071111 (2007).

${ }^{11}$ C.-C. Wu, C.-L. Lin, P.-Y. Hsieh, and H.-H. Chiang, Appl. Phys. Lett. 84, 3966 (2004).

${ }^{12}$ C.-W. Chen, C.-L. Lin, and C.-C. Wu, Appl. Phys. Lett. 85, 2469 (2004).

${ }^{13}$ T.-Y. Cho, C.-L. Lin, and C.-C. Wu, Appl. Phys. Lett. 88, 111106 (2006).

${ }^{14}$ J. Shi and C. W. Tang, Appl. Phys. Lett. 80, 3201 (2002).

${ }^{15}$ C.-H. Chang, Y.-J. Lu, C.-C. Liu, Y.-H. Yeh, and C.-C. Wu, J. Disp. Technol. 3, 193 (2007). 